\title{
Micromegas-TPC operation at high pressure in xenon-trimethylamine mixtures
}

\author{
S. Cebrián ${ }^{a}$, T. Dafni ${ }^{a}$, E. Ferrer-Ribas ${ }^{b}$, I. Giomataris ${ }^{b}$, D. Gonzalez-Diaz ${ }^{a, c}$, \\ H. Gómez ${ }^{a, d}$, D.C. Herrera ${ }^{a}{ }^{*}$, F.J. Iguaz ${ }^{a}$, I.G. Irastorza ${ }^{a}$, G. Luzon ${ }^{a}$, A. Rodríguez ${ }^{a}$, \\ L. Segui ${ }^{a}$, A. Tomás ${ }^{a}$ \\ ${ }^{a}$ Laboratorio de Física Nuclear y Astropartículas, Universidad de Zaragoza \\ C/ Pedro Cerbuna 12, 50009, Zaragoza, Spain \\ ${ }^{b}$ IRFU,Centre d'Etudes Nucleaires de Saclay (CEA-Saclay) \\ 91191 Gif sur Yvette, France \\ ${ }^{c}$ Department of Engineering Physics, Tsinghua University, Liuqing building, 10084, Beijing, \\ China \\ ${ }^{d}$ Laboratoire de l'Accélérateur Linéaire (LAL), Université Paris-Sud 11, Bâtiment 20091898 \\ Orsay Cedex (FRANCE) \\ E-mail: diana.heeunizar.es
}

\begin{abstract}
In this work we present a systematic study of Micromegas detectors in high pressure gaseous Xenon using trimethylamine (TMA) as quencher gas. Gas gains and energy resolutions for $22.1 \mathrm{keV} \mathrm{X-rays} \mathrm{are} \mathrm{measured} \mathrm{for} \mathrm{pressures} \mathrm{between} 1$ and $10 \mathrm{bar}$ and various relative concentrations of TMA from $0.3 \%$ to $15 \%$. We observe stable operation at all pressures, and a strongly enhanced gas gain, suggestive of Penning-like energy-transfer processes. The effect is present at all pressures and it is strongest at TMA concentrations ranging from $1.5 \%$ to $3 \%$. Operating in this concentration range, the maximum gain reached values as high as $\sim 2 \times 10^{3}\left(\sim 5 \times 10^{2}\right)$ at $1(10)$ bar. Besides, the energy resolution achievable for $22.1 \mathrm{keV} \mathrm{X-rays} \mathrm{is} \mathrm{substantially} \mathrm{better} \mathrm{than} \mathrm{the}$ one previously obtained in pure Xe, going down to $7.3 \%$ (9.6 \%) FWHM for 1 (10) bar. These results are of interest for calorimetric applications of high pressure gas Xe TPCs, in particular for the search of the neutrinoless double beta decay of ${ }^{136} \mathrm{Xe}$. The resolutions achieved would extrapolate into $0.7 \%(0.9 \%)$ FWHM at the $Q_{\beta}$ value of ${ }^{136}$ Xe for 1 (10) bar.
\end{abstract}

KeYwORDS: Micromegas; double beta decay; Time Projection Chambers; Penning effect.

${ }^{*}$ Corresponding autor 


\section{Contents}

1. Introduction 目

2. Experimental setup 2

3. Experimental procedure and method of analysis

4. Results and discussion

4.1] Optimum concentration of TMA

4.2 Varying the pressure

5. Conclusions 15

\section{Introduction}

Xenon-filled gaseous Time Projection Chambers (TPCs) are considered an interesting alternative to liquid Xe detectors in applications for which enhanced topological information, as well as energy resolution, are required. They have found successful applications, e.g., in X-rays astronomy and in double beta decay searches $(\beta \beta)$. The approach of using gas TPCs in $\beta \beta$ searches was pioneered by the Gothard TPC in the 1990's [四]. Much more recently, the NEXT experiment is following a similar approach and is constructing a $100 \mathrm{~kg}$ gas Xe TPC for this goal [2]. The NEXT readout concept relies on the detection of the scintillation of pure Xe for both $t_{0}$ determination and enhanced energy resolution. Another collaboration, EXO-gas, also develops a gas TPC to search for the double beta decay of ${ }^{136} \mathrm{Xe}$, and considers several options for its readout [3].

More generically, the technical complexity of gas TPCs has been partially alleviated by the advent of micropattern gas detectors (MPGD), simpler and with better scaling-up prospects than conventional multiwire proportional chambers (MWPC). One of the most attractive MPGD from the point of view of energy resolution are the Micromesh Gas Structures (Micromegas) [四]. These readouts are object of active development for application to rare event searches within the context of the R\&D project T-REX [5]. In particular, Micromegas fabricated with the microbulk technique [6] have shown particularly good prospects regarding aspects like radiopurity [प], or energy resolution [ [8], both aspects crucial for application to $\beta \beta$ searches. The results presented in this paper are a further step in the studies of energy resolution with this type of readouts.

Micromegas readouts make use of a metallic micromesh suspended over an anode plane by means of insulator pillars, defining an amplification gap of the order of 50-150 $\mu \mathrm{m}$. It is known [Q] that the way the amplification develops in a Micromegas gap is such that its gain $M$ is less dependent on geometrical factors (the gap size) or environmental ones (like the temperature or pressure of the gas) than other MPGDs or MWPCs. In addition, the amplification in the Micromegas gap has 
less inherent statistical fluctuations than that of wires, due to the faster transition from the drift field to the amplification field provided by the micromesh [10]. Micromegas of the microbulk type are built out of a double-clad kapton foil, by applying an appropriate chemical bath that removes both copper and kapton to form the Micromegas holes [1]]. The homogeneity of the gap in these structures is superior to other more conventional Micromegas, further improving gain stability and homogeneity and therefore energy resolution. Experimentally, Full Width at Half Maximum (FWHM) resolutions of $\sim 11 \%$ for the $5.9 \mathrm{keV}$ peak of ${ }^{55} \mathrm{Fe}$ in Ar-based mixtures are now routinely achieved by the latest generation of these detectors.

The work performed up to now has been focused on establishing the capability of microbulk detectors to work in high pressure pure $\mathrm{Xe}$, and more specifically to measure their energy resolution in those conditions. In our previous works [12] we have experimentally demonstrated that operation of microbulk Micromegas in pure noble gases is feasible, even at high pressures, and reaching gains above 100 for the case of Xe. This is a remarkable result because in absence of quencher early breakdown occurs at high pressure in all other MPGDs [13]. We tentatively attribute this result to the "geometrical quenching" achieved by the fact that the avalanche in microbulk detectors happens totally enclosed in a kapton cell, preventing to some extent the propagation of photondriven avalanches. Geometrical streamer-quenching is indeed a documented phenomenon, and has been used with limited success in some non-standard implementations of Resistive Plate Chambers [14].

In this work we present results obtained with trimethylamine (TMA) as a quencher for Xe. The mixture Xe + TMA was studied in the past for wire chambers at 1 bar [15]. The authors of [15] have shown that the addition of TMA to Xe at a given field, substantially increases the detector gain with respect to the one in pure Xe, a phenomenon attributed to Penning effect. On the other hand, improvements of $\sim 25 \%$ in energy resolution have also also observed compared with the conventional non-Penning mixtures. We extend here that study to higher pressures up to 10 bar using Micromegas detectors. We perform systematic measurements of gas gain and energy resolution for different TMA fractions, from $0.3 \%$ to $15 \%$. In general, the operation of the detector in Xe+TMA mixtures improves substantially the gas gain as well as the energy resolution, up to concentrations of the quencher in the range $\sim 2-3 \%$ and for all pressures tested. The improvement is specially outstanding at high pressures, given that operation in pure Xe degrades rapidly with pressure [13]. We believe that this systematic study could provide a starting point for evaluating the recent proposal of using TMA as a means to reduce the Fano factor in Xe-based chambers [16].

The article is structured as follows: in section 2 we describe the experimental setup and in section 3 the experimental procedure. In section 4 we present the results obtained and discuss them. We finish in section 5 with our conclusions.

\section{Experimental setup}

The experimental setup used in the present investigation is similar to the one used in our earlier studies in pure Xe and has been described in detail in a previous paper [12]. It consists of a cylindrical stainless steel chamber and a gas system. The gas system includes: a filling gas line, 
a recirculation gas line, a gas recovery line and a mass spectrometer line. The last two parts have been added to the previous setup. A schematic view is shown in figure 1 .

The chamber has an active volume of 2.2 liters (10 cm in height, $16 \mathrm{~cm}$ in diameter) constructed with ultra-high vacuum specifications and is rated for operation at 15 bar. Inside the chamber, two circular copper plates $(10 \mathrm{~cm}$ in diameter) form the drift/conversion region of $1 \mathrm{~cm}$. The cathode (top plate) is connected to negative voltage. Centered relative to the bottom plate, a Micromegas is located, having $35 \mathrm{~mm}$ diameter, $50 \mu \mathrm{m}$ gap, with $50 \mu \mathrm{m}$ diameter holes and $115 \mu \mathrm{m}$ pitch. The detector was built with the microbulk technique [6]. The mesh is grounded to the body chamber while a positive voltage is applied to the anode. The signal from the anode is fed into a CANBERRA preamplifier+amplifier chain (model 2005 and 2022 respectively), the latter with a shaping time of $4 \mu \mathrm{s}$. The amplified signal is finally recorded by a multichannel analyzer (AMPTEK MCA 8000A), that produces the pulse height distribution.

The gas filling line and recirculation line, including a membrane pump (circulating process), several valves, gauge pressures, and filters are sketched in detail in figure 1. In addition, a new purifier has been installed (SAES 702), which removes water vapor and electronegative impurities $\left(\mathrm{H}_{2} \mathrm{O}, \mathrm{O}_{2}, \mathrm{CO}_{2}\right)$ and it is compatible with TMA. The vacuum system includes a turbo-molecular pump, two gauge pressures and four outlets that permit to attain vacuum values down to $10^{-6}$ mbar.

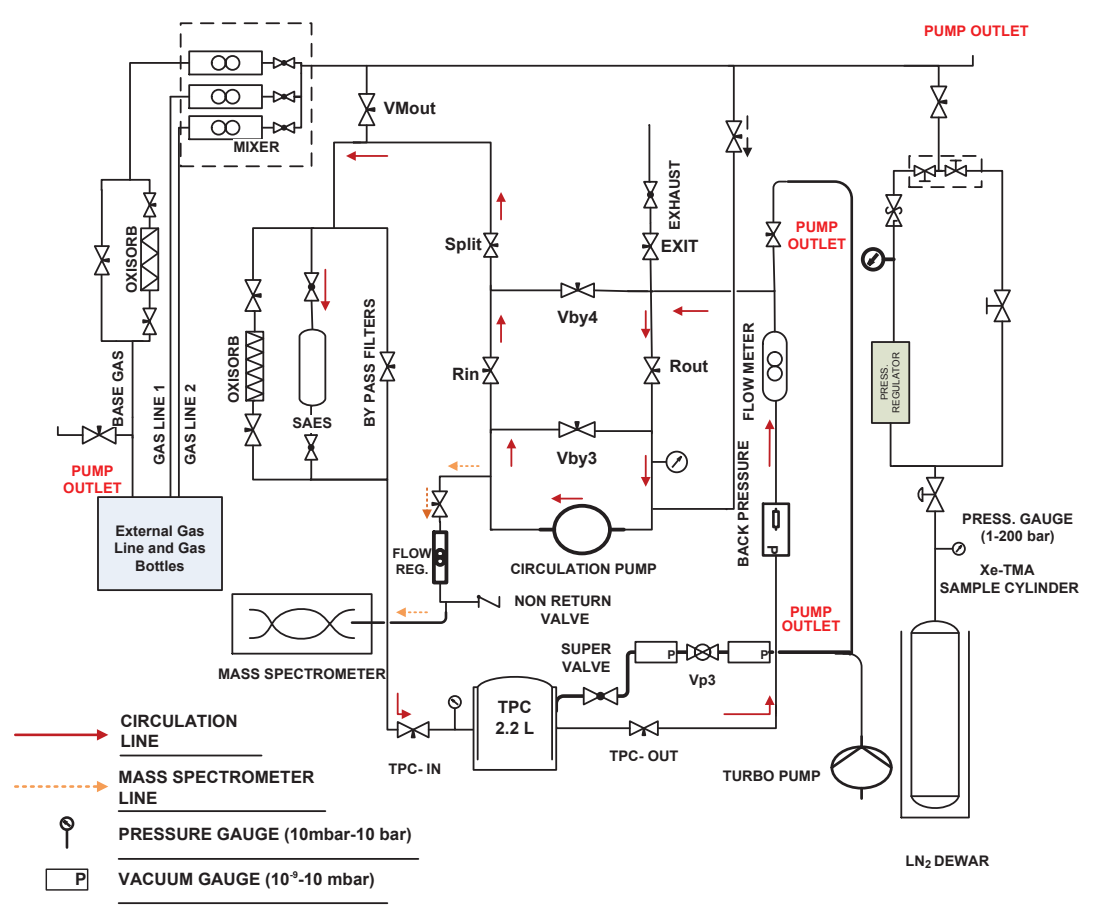

Figure 1. Schematic view of the experimental setup with the location of the TPC, the Xe-TMA sample cylinder and the vacuum, filling, purification, recovery and mass spectrometer lines.

The gas recovery system has a stainless steel sample cylinder of 2.2 liters that is immersed in a Dewar flask filled with liquid nitrogen $\left(\mathrm{LN}_{2}\right)$ during the recovery process. The outlet of the sample cylinder is connected to two gas lines: one of them for the gas recovery and the other one 
for the filling of the TPC. On the other hand, a Pfeiffer OmniStar mass spectrometer has been added to the gas system via a low pressure gas line. The mass spectrometer is used to quantify the gas composition of Xe+TMA mixtures and to monitor the electronegative impurities. Measurements filling the gas system at 2 bar with a pre-mixed $75 \% \mathrm{Xe}+25 \%$ TMA mixture were performed in order to establish the calibration factor needed for determining the amount of TMA in each mixture used. In case of water vapor and oxygen only relative changes could be estimated.

\section{Experimental procedure and method of analysis}

All measurements were carried out with a ${ }^{109} \mathrm{Cd}$ source collimated with a $2 \mathrm{~mm}$ diameter spot, that was placed on the center of the cathode plate. The chamber and the gas system were pumped to values down to $10^{-6}$ mbar. The chamber was routinely baked out at a temperature of $100^{\circ} \mathrm{C}$ during three hours, obtaining outgassing rates below $5 \times 10^{-5} \mathrm{mbarl} / \mathrm{s}$, before the gas filling. The Xe used had a purity-grade of 6 . At the beginning of all measurements, a Xe+TMA mixture with high concentration $(\sim 7 \%)$ of TMA was prepared in the sample cylinder. Lower concentrations of the additive gas were obtained adding fresh Xe from the external bottle. After the preparation of every new mixture, we observed that the circulation of the mixture through the filter produced changes in the TMA concentration, that is due to the SAES filter absorbing or expelling TMA depending on the previous gas mixture used. This fact forced us to monitor the TMA concentration obtained after a certain stabilization time, that was never exceeding $1 \mathrm{~h}$. The procedure was as follows: the chamber was filled at the desired pressure, and the circulation process through the purifier started, keeping the pressure constant. Secondly, energy spectra were acquired every two minutes at a gas gain above 100 and a reduced drift field below $100 \mathrm{~V} / \mathrm{cm} / \mathrm{bar}$. Then the gas gain and energy resolution were monitored until the variations seen were less than $5 \%$. Such stationary conditions were typically reached within some 20 minutes, a situation that we interpret as the mixture being homogeneous throughout the complete gas system. This fact was verified several times, monitoring the TMA concentration with the mass spectrometer at the same time.

Once the gas mixture was stabilized, the operating point was established by ensuring the maximum electron transmission of the Micromegas mesh. For this goal the amplification voltage was kept constant for a gas gain around 300 while the drift voltage was scanned within a range 0.1-1 $\mathrm{kV} / \mathrm{cm}(1 \mathrm{bar})$ and $0.2-5 \mathrm{kV} / \mathrm{cm}(10 \mathrm{bar})$. Figure 2 shows the typical curves of electron transmission as a function of the ratio between drift and amplification fields for pressures from 1 to $10 \mathrm{bar}$ using mixtures with around $1.5 \%$ TMA, where the values have been normalized to the maximum of each curve. Since the electron transmission depends on the ratio of drift-to-amplification fields

$E_{\text {drift }} / E_{\text {amp }}$, as it is showed in figure 2 and in earlier works [四, [1], it was kept constant during measurements.

The increase in the amplification field was stopped when consecutive sparks occurred in a short time (30 s), typically the variations were between 34 (84) and 58 (114) kV/cm for 1 (10) bar. In particular, at high pressures where it is necessary to apply high fields, the increase was until a spark occurred in order to avoid irreversible damage. At the end of each set of measurements, the TMA concentration was measured with the mass spectrometer. Finally, the chamber and the gas system was cleaned by recovering the gas and then pumping it. The recovery process was performed by cooling the sample cylinder to $\mathrm{LN}_{2}$ temperature (11). 


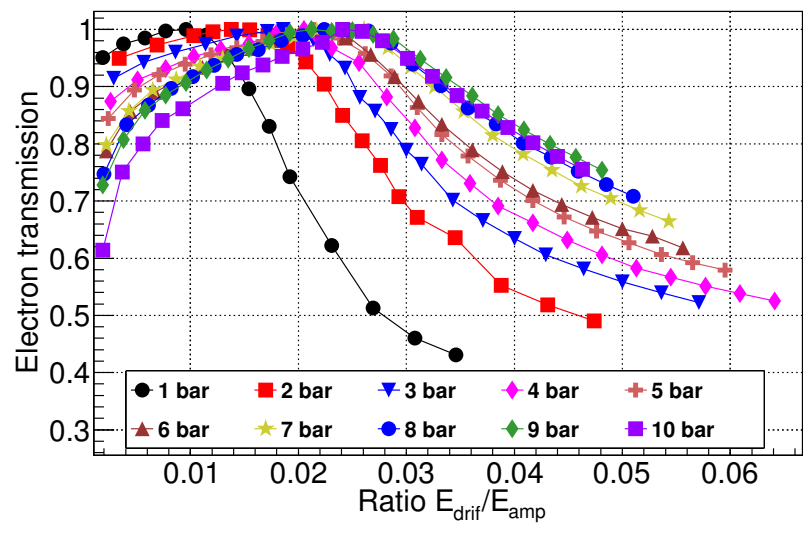

Figure 2. Electron transmission of the Micromegas detector as a function of the ratio of drift-to-amplification fields in Xe+TMA mixtures, for pressures between 1 and 10 bar. The percentage of TMA for each pressure is around $1.5 \%$. The operation point was selected within the plateau region.

In the offline analysis, the peak at $22.1 \mathrm{keV}$ was used to obtain the gas gain and energy resolution. As the mean energy for the formation of an ion pair $(\mathrm{W})$ has not been measured for Xe+TMA mixtures, the value of $22 \mathrm{eV}$ for pure $\mathrm{Xe}$ was used for all gas gains calculations; the estimated error due to this assumption is below $10 \%$. In figure 3, the typical energy spectrum acquired with a ${ }^{109} \mathrm{Cd}$ source is shown at 1 bar in a $\mathrm{Xe}+1.7 \%$ TMA mixture (left) and at 10 bar with $1.1 \%$ TMA (right). In both spectra the $\mathrm{K}_{\alpha}$ and $\mathrm{K}_{\beta}$ lines from Ag fluorescence are clearly distinguished. The corresponding escape peaks from $\mathrm{Xe}$ are observed below the $\mathrm{K}_{\alpha}$ line, located at $17.9 \mathrm{keV}$ and 20.8 $\mathrm{keV}$. The $\mathrm{Cu} \mathrm{K}$-fluorescence at $8.1 \mathrm{keV}$ is also observed in the spectra. These events are produced from the interaction of X-rays with the electrodes of the Micromegas.
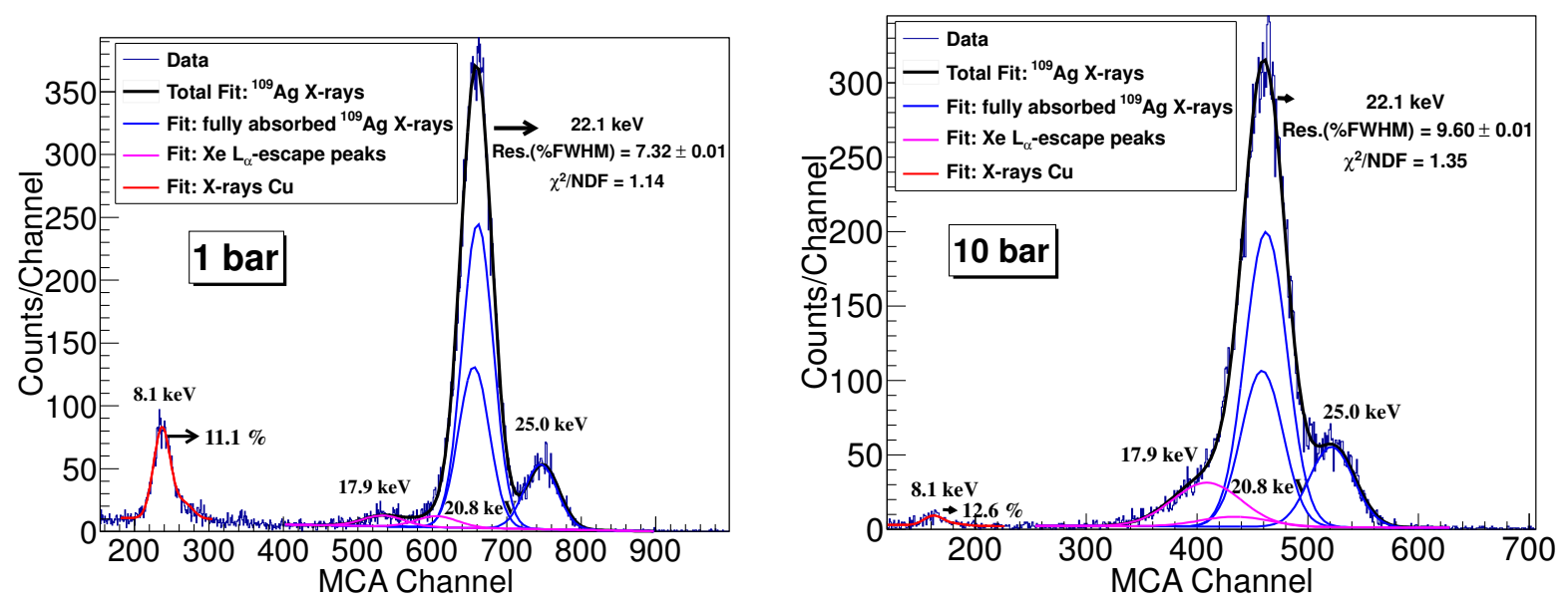

Figure 3. Characteristic X-rays energy spectra originated from ${ }^{109} \mathrm{Cd}$ source, in a $\mathrm{Xe}+1.7 \%$ TMA mixture at 1 bar (left) and $1.1 \%$ TMA at 10 bar (right). The fit performed to the overall ${ }^{109} \mathrm{Ag} \mathrm{K}$-fluorescence lines consists in a 3-step routine that is shown in each spectrum. In addition, the $\mathrm{Cu}$ K-fluorescence at $8.1 \mathrm{keV}$ is separately fitted to a single Gaussian. 
The $\mathrm{Ag} \mathrm{K} \mathrm{K}_{\alpha}, \mathrm{K}_{\beta}$ lines and the corresponding Xe escape peaks (L-shell) were fitted in the energy range between 14 and $30 \mathrm{keV}$ using a 3-step routine. The results of this procedure are shown in figure 3. In the first step, the complete range was fitted to one Gaussian function $\mathrm{K}_{\alpha 1}(22.2 \mathrm{keV})$ over a linear background. In the second one, two Gaussian functions were added corresponding to $\mathrm{K}_{\alpha_{2}}(22.0 \mathrm{keV})$ and $\mathrm{K}_{\beta}(25.0 \mathrm{keV})$ lines. In the last step, two more Gaussian functions were added which correspond to the Xe X-rays escape peaks. The input parameters in the last two steps are the calculated ones at previous steps.

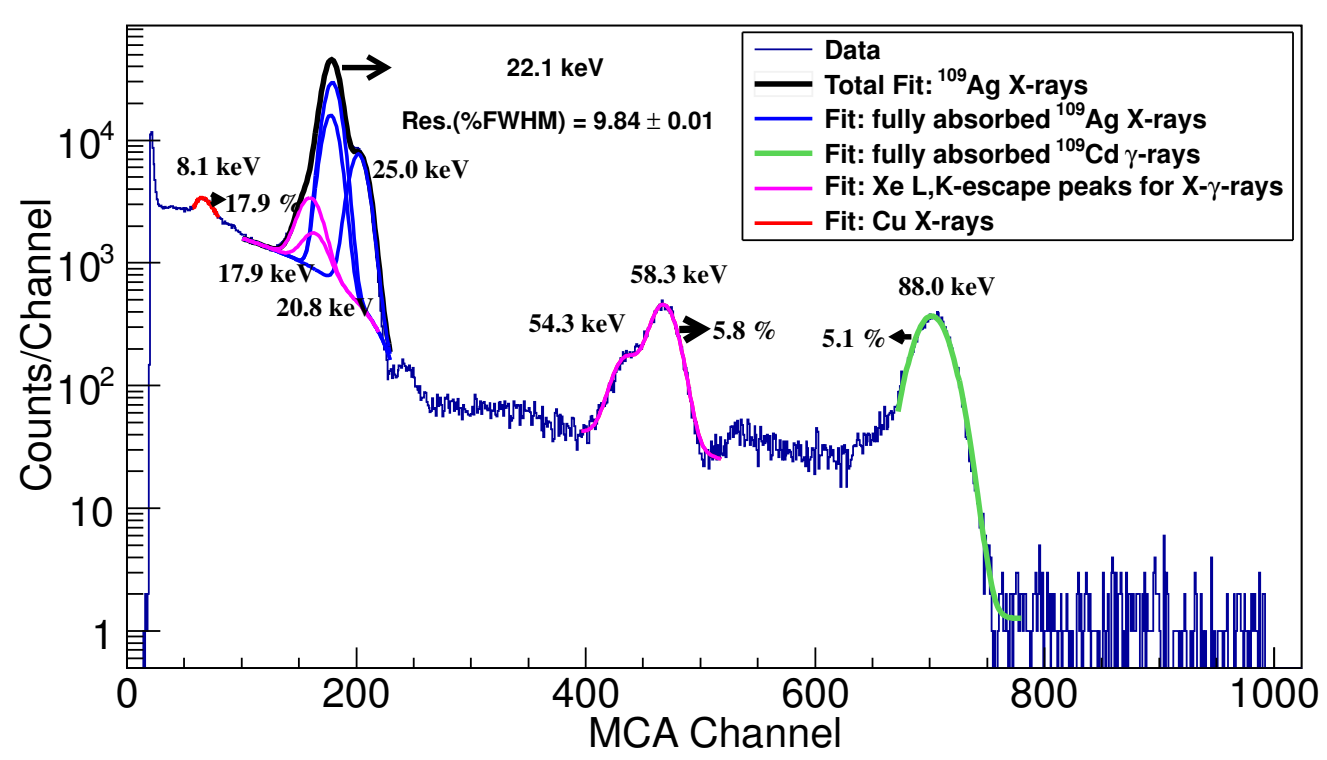

Figure 4. Energy spectrum from a ${ }^{109} \mathrm{Cd}$ source acquired at 8 bar in a $\mathrm{Xe}+1.4 \%$ TMA mixture, with $E / p=245 \mathrm{~V} / \mathrm{cm} / \mathrm{bar}$. The $\mathrm{K}$-fluorescence lines from ${ }^{109} \mathrm{Ag}$ with their respective escape peaks of Xe are shown. The $\gamma$-line from ${ }^{109} \mathrm{Cd}$ at $88.0 \mathrm{keV}$ is observed as well as the escape peak at $58.3 \mathrm{keV}$. The fits realized for each peak and the energy resolution (\%FWHM) are depicted on the spectrum.

Figure 4 shows an energy spectrum acquired at 8 bar using a mixture of $\mathrm{Xe}+1.4 \%$ TMA, with a larger energy range than previous figure. It is interesting to observe the $\gamma$-rays from ${ }^{109} \mathrm{Cd}$ at 88.04 $\mathrm{keV}$ and two escape peaks located at $58.3 \mathrm{keV}$ and $54.3 \mathrm{keV}$, which correspond to $\mathrm{K}_{\alpha}(29.7 \mathrm{keV})$ and $\mathrm{K}_{\beta}(33.7 \mathrm{keV})$ of $\mathrm{Xe}$, respectively. The energy resolution of the main peaks is depicted, with values of 9.8, 5.8, 5.1\% FWHM at 22.1, 58.3 and $88.0 \mathrm{keV}$, respectively. As expected, the energy resolution shows a dependency with the inverse of the square-root of the energy. We must point out that the energy spectrum in figure 4 was not acquired under optimum fields for this mixture and the only interest was to observe the $\gamma$-rays of ${ }^{109} \mathrm{Cd}$. The event containment and conversion probability of ${ }^{109} \mathrm{Cd} \gamma$-rays is indeed only well suited for the highest pressures, making their study at low/medium presssure more complicated in our setup.

\section{Results and discussion}

The first goal of this work was to establish a range of TMA concentration in which it would be possible to obtain the best energy resolution and highest gain for pressures between 1 and 10 bar. 
Hence, a systematic variation of the TMA concentrations was performed at four reference pressures: 1, 5, 8 and 10 bar and results are presented in section 4.1. An optimal range of TMA concentration was thus estimated, in which Penning-like transfer processes from excited Xe states are maximized. Once the optimum concentration range was found, we performed a systematic study varying the pressure between 1 and $10 \mathrm{bar}$, which is presented in section 4.2 .

\subsection{Optimum concentration of TMA}

The variation in TMA concentration was performed at four pressures in different ranges: at 1 bar $(0.4 \%-15.5 \%), 2$ bar $(0.4 \%-6.0 \%)$, at 8 bar $(0.3 \%-5.0 \%)$, and 10 bar $(0.8 \%-6.2 \%)$. The active response of the filter to TMA led in practice to slightly different families of mixtures for each pressure. The optimum range of TMA concentration was selected based on the dependences of the gain and the energy resolution with the amplification field, as described below.

\subsubsection{Gas gain}

The dependence of the gain with the amplification field is shown in figure 5 at four pressures: 1 (a), 5 (b) , 8 (c), and 10 (d) bar. The gain curves show a linear behaviour with the amplification field in the semi-log plot, having correlation factors above 0.999 except for special cases with low statistics.

Considering the plot at 1 bar (see figure 5a), we observe that lower amplification fields must be applied when the TMA percentage is increased from $0.4 \%$ to $1.4 \%$. The curves within a concentration range from $1.4 \%$ and $6.4 \%$ TMA seem to overlap, suggesting that transfer mechanisms are already fully active while the avalanche dynamics remains largely unaffected. The tendency reverses above $6.4 \%$ TMA so that higher fields must be applied to obtain the same gas gain, as also observed in neon-based mixtures [17]. A similar behaviour is observed at high pressures (see figure $5 \mathrm{~b}-\mathrm{d}$ ). This fact is more clear at high pressures, when low quenched mixtures are compared with highly quenched ones, being the trend also present at 1 bar, but less pronounced.

In order to do a better study of the optimum TMA concentration, we performed linear fits of the $\ln G$ versus amplification field data for proper interpolation or extrapolation of the gain or field. For instance, figure 6 (left) shows the dependence of the amplification field calculated to obtain a gas gain of 300 versus the TMA concentration at each pressure. If we consider the points at 1 bar, by increasing the TMA concentration the field needed to obtain the same gain is reduced down to $44 \mathrm{kV} / \mathrm{cm}$ for $1.7 \%$ TMA. This field remains practically constant between $1.4 \%$ and $6.4 \%$ TMA, increasing at higher concentrations. The same dependency is observed at high pressures: the amplification field reaches a minimum value at around $2 \% \mathrm{TMA}$, being $75,95,109 \mathrm{kV} / \mathrm{cm}$ at 5,8 and 10 bar, respectively.

On the other hand, representing the variation of the gas gain with the percentage of TMA is another way to see that an optimum TMA concentration is reached at each pressure, for which the influence of transfer processes is maximized. The minimum values of the amplification field reached before for gas gains of 300, were used to study the behaviour of the gain (at each pressure) as a function of the TMA concentration (see figure Gb)(right). The gain rapidly increases when small quantities of TMA are added, increasing by a factor of 16 (50) at 1 (5) bar when TMA increases from $0.4 \%$ to $2 \%$. The rapid rise in gas gain at constant amplification field has also been observed using $\mathrm{Xe}+2,3$ dimethyl-2-butene as Penning additive, showing similar dependences with 

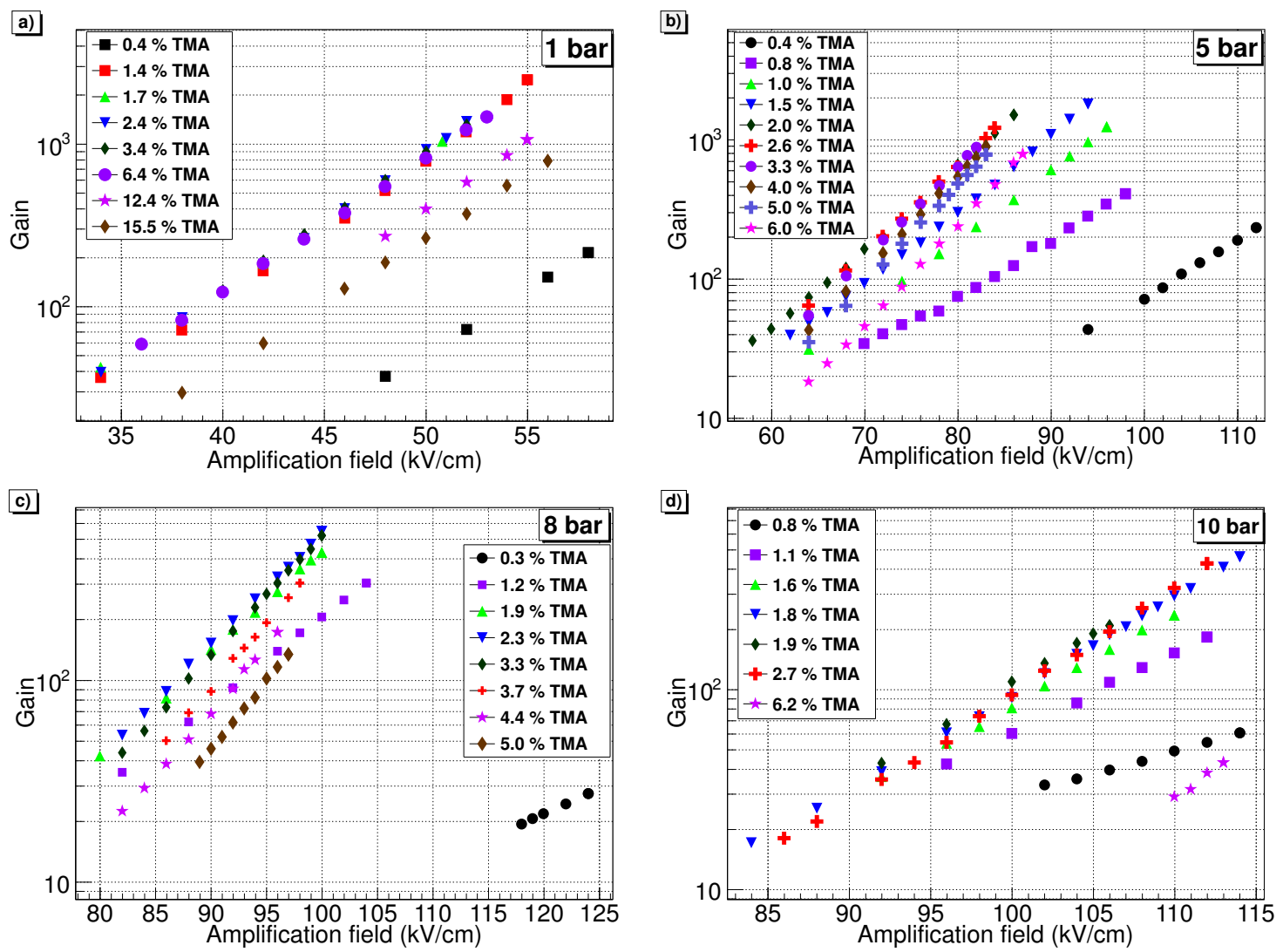

Figure 5. Dependency of gas gain with amplification field for different TMA concentrations at 1 (a), 5 (b), 8 (c), 10 (d) bar. In each graph the TMA concentration is indicated.

the additive at 1 bar [18]. Even stronger changes are seen at 8 and 10 bar. This great increase is a strong evidence that Penning effect takes place, being stronger at high pressure. In general, the gas gain reaches a maximum value within the range of $2 \%-2.5 \%$, then it drops.
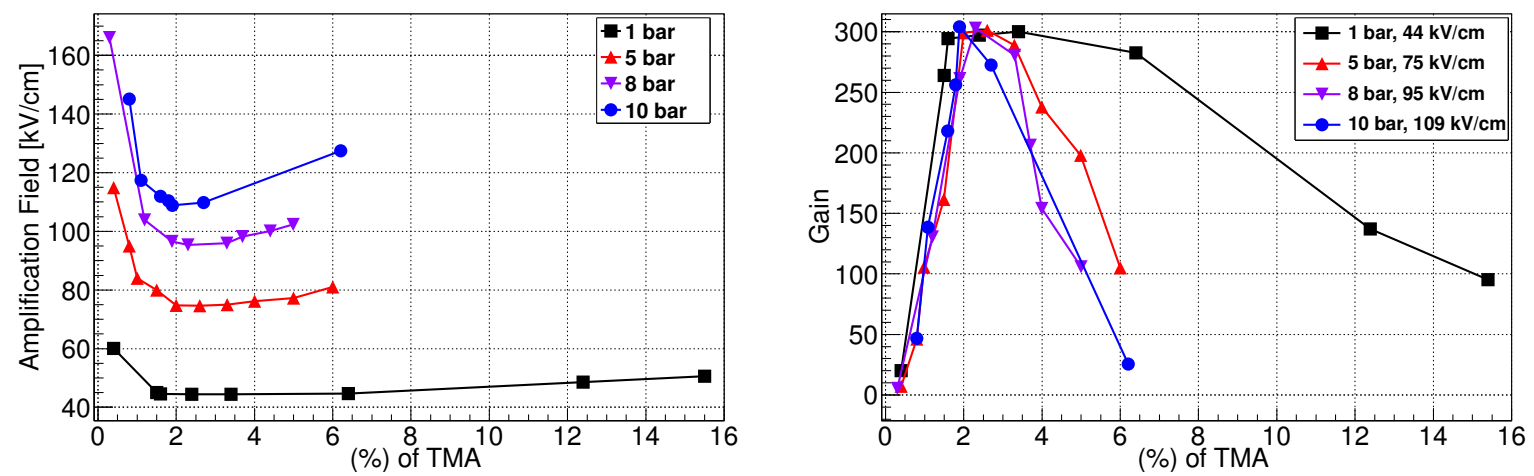

Figure 6. Left: Amplification field needed for a gas gain of $G=300$ as a function of the TMA percentage. Right: Gas gain as a function of the TMA percentage at a fixed pressure and amplification field. For each data set, an optimum for the the Penning effect can be clearly observed. 
The onset of breakdown at each pressure presents a similar behaviour: by increasing the TMA concentration, the maximum gas gain increases up to maximal values within a TMA concentration range of $2 \%$ to $3 \%$; above these values, the maximum gain falls. Values of maximum gas gain below 60 are obtained for TMA concentrations less than 1\%, while within optimum range values gains above 2000 (400) at 1 (10) bar have been attained. This fact is an important advantage in comparison with pure $\mathrm{Xe}$, because it permits to work at higher gas gain with lower amplification fields in stable conditions. In some cases this trend was broken, attributed mainly to dust on the surface of the detector, which can limit the maximum gain, specially at high pressures. Hence, the detector was routinely washed and then flushed with helium, reaching higher amplifications fields than before.

\subsubsection{Energy Resolution}

The dependence of the energy resolution with the gas gain for $22.1 \mathrm{keV} \mathrm{X-rays} \mathrm{is} \mathrm{shown} \mathrm{in} \mathrm{figure}$ 7 for four pressures: 1 (a), 5 (b), 8 (c) and 10 bar (d). The energy resolution improves as the gas gain increases for all mixtures. The worse values at low gains are due to electronic noise, so its contribution was measured at FWHM injecting at the amplifier input an AC-coupled square pulse, and subtracting its value in quadrature. It was found that the resulting energy resolution has compatible values in the gas gain range between 50 and 900 . This allows to estimate that the noise contribution for gas gains above 300 is less than 1\%. Hereafter, all values of resolution presented or plotted in this paper include the noise contribution. For Xe+TMA mixtures within the optimal range for Penning transfer of $1.5 \%-3 \%$, the energy resolution degrades at the highest gas gain, more slowly at 5 bar than at 1 bar. However, this deterioration was not observed at high pressures ( 8 and 10 bar) since the maximum gas gain was below 500. Previous authors [19] have suggested that this deterioration is caused by space charge effects, despite the small gains involved.

In table 1 the best values obtained for the energy resolution are listed together with the gas gain at which they were measured. At each pressure, the energy resolution improves adding TMA, taking minimum values around 2\% TMA and deteriorating at higher concentrations, At 1 bar, however, the minimum is not observed. For instance, at 1 bar, the energy resolution goes from a value of $8.4 \%$ (0.4\% TMA) down to $7.3 \%$ FWHM at $22.1 \mathrm{keV}$ (1.7\% TMA), then it remains constant between $1.4 \%$ and $15.4 \%$, for gains above 800 . These values of the energy resolution are compatible with measurements performed on a cylindrical proportional counter using $95 \% \mathrm{Xe}+$ 5\% TMA at 1 bar [15]. On the other hand, at high pressures the energy resolution deteriorates at even lower TMA percentages, the range where compatible values are obtained being $\sim 1.0 \%-2.6 \%$. The best energy resolution is $8.3 \%, 9.0 \%$ and $9.6 \%$ FWHM for 5, 8 and 10 bar, respectively. It must be noted, that we observe more variations in energy resolution within this optimum range, which are more pronounced with pressure. We estimated our level of impurities below $30 \mathrm{ppm}$ for all mixtures, based on the relative variations determined with the mass spectrometer and considering that our values for the energy resolution are compatible with those quoted in [19].

As a summary, we can infer from these results that the best energy resolution can be obtained using TMA fractions between $1 \%$ and $2.5 \%$, while in the previous section we have seen that the maximum gas gains are attained in a range of $2 \%$ to $3 \%$ TMA. Therefore, we can conclude that optimal Xe+TMA mixtures should be ranging from $1.5 \%$ to $2.5 \%$. For applications where the energy resolution is the most important factor, TMA concentrations above $1 \%$ should be used. 


\begin{tabular}{|c|c|c|c|c|}
\hline$\%$ TMA & Best Ene. Res. (\% FWHM) & Gain $\left[\times 10^{2}\right]$ & $A\left(\mathrm{~cm}^{-1} \mathrm{bar}^{-1}\right)$ & $B(\mathrm{kV} / \mathrm{cm} / \mathrm{bar})$ \\
\hline \multicolumn{5}{|c|}{ Pressure 1 bar } \\
\hline 0.4 & 8.4 & 1.5 & $6986 \pm 284$ & $108.9 \pm 2.0$ \\
\hline 1.4 & 7.4 & 5.2 & $5007 \pm 251$ & $66.7 \pm 2.1$ \\
\hline 1.7 & 7.3 & 8.6 & $4507 \pm 96$ & $61.1 \pm 0.8$ \\
\hline 2.4 & 7.3 & 9.3 & $5073 \pm 167$ & $66.3 \pm 1.4$ \\
\hline 3.4 & 7.5 & 9.0 & $5058 \pm 172$ & $66.1 \pm 1.4$ \\
\hline 6.4 & 7.3 & 8.2 & $4974 \pm 153$ & $65.7 \pm 1.3$ \\
\hline 12.4 & 7.3 & 5.8 & $6020 \pm 672$ & $80.8 \pm 5.6$ \\
\hline 15.5 & 7.3 & 5.5 & $5714 \pm 304$ & $81.7 \pm 2.5$ \\
\hline \multicolumn{5}{|c|}{ Pressure 5 bar } \\
\hline 0.4 & 8.9 & 2.3 & $1665 \pm 81$ & $45.5 \pm 1.0$ \\
\hline 0.8 & 8.6 & 4.1 & $988 \pm 16$ & $27.9 \pm 0.3$ \\
\hline 1.0 & 8.4 & 7.3 & $1182 \pm 46$ & $27.6 \pm 0.6$ \\
\hline 1.5 & 8.3 & 8.1 & $1144 \pm 46$ & $25.8 \pm 0.6$ \\
\hline 2.0 & 8.4 & 6.5 & $1228 \pm 35$ & $25.2 \pm 0.4$ \\
\hline 2.6 & 8.5 & 6.4 & $1492 \pm 23$ & $28.1 \pm 0.2$ \\
\hline 3.3 & 8.9 & 8.8 & $1772 \pm 39$ & $30.7 \pm 0.3$ \\
\hline 4.0 & 9.0 & 7.4 & $2008 \pm 38$ & $33.1 \pm 0.3$ \\
\hline 5.0 & 9.0 & 7.8 & $2246 \pm 43$ & $35.3 \pm 0.3$ \\
\hline 6.0 & 9.6 & 7.9 & $2783 \pm 15$ & $40.6 \pm 0.1$ \\
\hline \multicolumn{5}{|c|}{ Pressure 8 bar } \\
\hline 0.3 & 27.3 & 0.2 & $708 \pm 79$ & $33.3 \pm 1.7$ \\
\hline 1.2 & 9.1 & 3.0 & $809 \pm 58$ & $22.7 \pm 0.8$ \\
\hline 1.9 & 9.0 & 3.6 & $1162 \pm 99$ & $25.3 \pm 1.0$ \\
\hline 2.3 & 9.4 & 5.4 & $1379 \pm 42$ & $27.0 \pm 0.3$ \\
\hline 3.3 & 9.8 & 4.5 & $1696 \pm 41$ & $29.6 \pm 0.3$ \\
\hline 3.7 & 10.4 & 3.0 & $2224 \pm 90$ & $33.6 \pm 0.5$ \\
\hline 4.4 & 11.4 & 1.7 & $2519 \pm 59$ & $35.7 \pm 0.3$ \\
\hline 5.0 & 12.0 & 1.3 & $3399 \pm 222$ & $40.2 \pm 0.8$ \\
\hline \multicolumn{5}{|c|}{ Pressure 10 bar } \\
\hline 0.8 & 19.7 & 0.6 & $330 \pm 25$ & $15.9 \pm 0.8$ \\
\hline 1.1 & 9.6 & 1.8 & $772 \pm 43$ & $22.4 \pm 0.6$ \\
\hline 1.6 & 10.2 & 2.3 & $997 \pm 45$ & $24.3 \pm 0.5$ \\
\hline 1.8 & 10.2 & 4.6 & $1072 \pm 13$ & $24.7 \pm 0.1$ \\
\hline 1.9 & 10.1 & 2.0 & $1141 \pm 54$ & $25.0 \pm 0.5$ \\
\hline 2.7 & 10.4 & 4.2 & $1423 \pm 29$ & $27.6 \pm 0.2$ \\
\hline 6.2 & 47.0 & 0.4 & $4644 \pm 1033$ & $46.6 \pm 4.7$ \\
\hline
\end{tabular}

Table 1. Values of the best energy resolution obtained at each TMA concentration for pressures of $1,5,8$ and $10 \mathrm{bar}$, with the corresponding value of gas gain at which it was obtained. The systematic error of all values is estimated to be less than $0.2 \%$ FWHM. Values of the parameters $A$ and $B$ together with their errors are obtained from the fit to the equation 4.1 . 

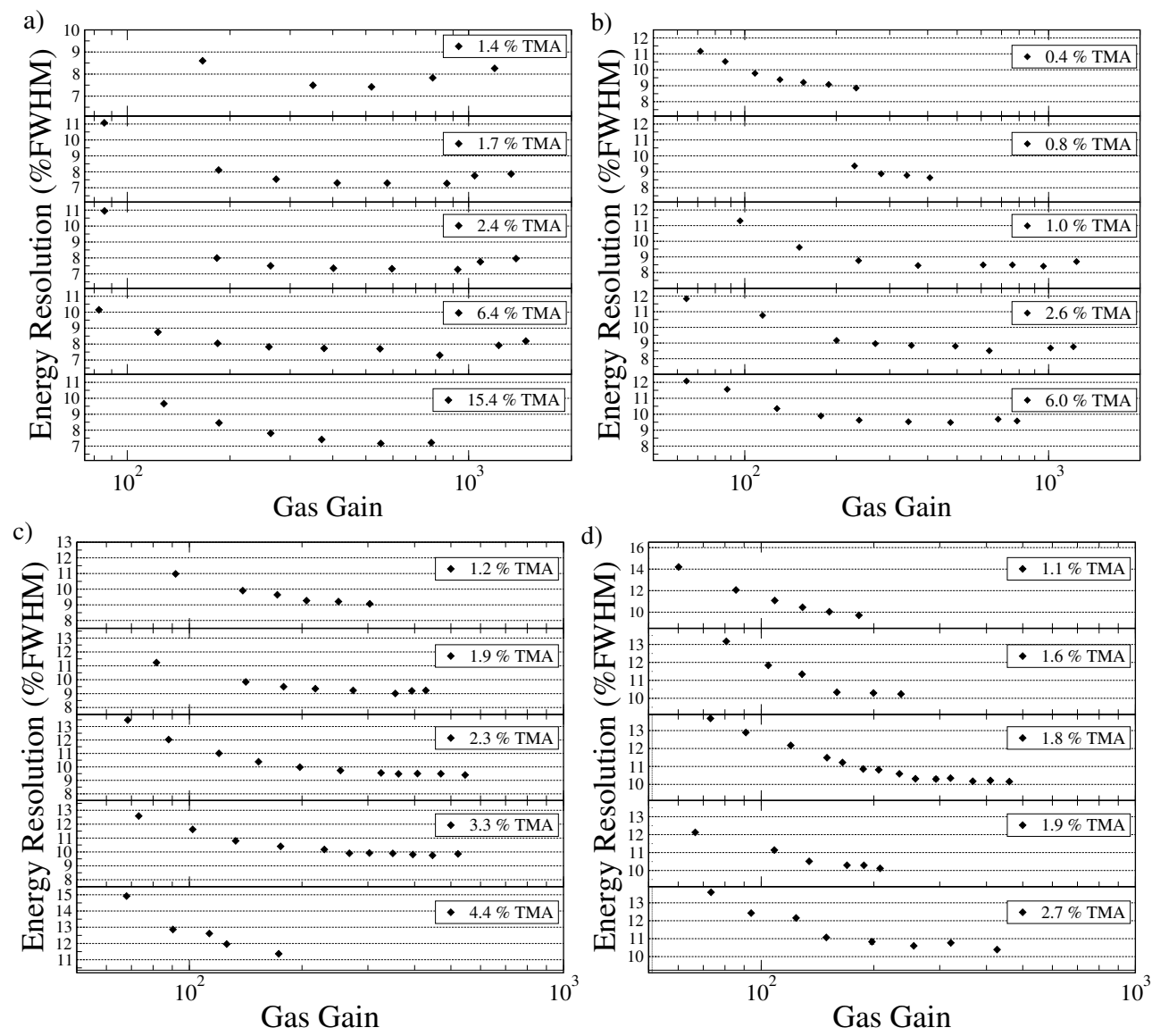

Figure 7. Energy resolution at $22.1 \mathrm{keV}$ as a function of gas gain for different TMA concentrations at 1 (a), 5 (b), 8 (c) and 10 bar (d). The TMA concentration is indicated for each data set.

\subsubsection{First Townsend parameter}

The first Townsend coefficient $(\alpha)$ permits to describe the electron multiplication processes in Micromegas. Taking into account a semi-empirical parameterization of this coefficient [20],

$$
\alpha=A P \exp (-B / S)
$$

where $S$ is the reduced amplification field $\left(S=E_{a m p} / P\right), P$ is the gas pressure, and $A$ and $B$ are constants that depend on the gas. The logarithm of the gas gain of a Micromegas is the integral of the Townsend coefficient along the field lines from the mesh to the anode. By assuming a constant field, in first order, the electron multiplication $G$ can be written as [21]:

$$
\ln \left(\frac{\ln (G)}{P}\right)=\ln (A d)-\frac{B}{S}
$$

where $d$ is mesh-anode distance and $A$ and $B$ can be obtained from a linear fit of the $\ln (\ln (G))$ vs. $S^{-1}$ data. According to this simplified physical picture quoted by the authors of [19, 22], $A$ and $B$ are related with microscopic parameters where: $A$ corresponds to the inverse of the collision mean free path at the reference pressure $P_{0}\left(A=1 /\left(\lambda_{0} P_{0}\right)\right)$, while $B=V_{i} /\left(\lambda_{0} P_{0}\right)$, where $V_{i}$ is the effective energy to produce an electron-ion pair. 
In table [1 the corresponding parameters for each set of data are shown. Under the physical picture in [19], the mean energy of the electron swarm between two collisions is given by $\bar{\varepsilon}=E \lambda=E \lambda_{0} P_{0} / P$. The ratio $\bar{\varepsilon} / V_{i}=E \lambda / V_{i}$ gives hence a figure on how likely ionization is to occur. Naturally, for large $\lambda / V_{i}$, ionization will occur at lower fields. This implies that $B \times P=V_{i} / \lambda$ can be seen as a characteristic reduced field at which the probability of ionization becomes important. Therefore, the following interpretation of the observations can be made:

- for small TMA concentrations $B$ starts from a given value, asymptotically approaching the one in pure Xenon.

- as TMA increases, Penning transfers become active, allowing for ionization to take place at lower fields. This process is mediated by excited atomic and molecular states, that require considerably less energy for being ionized than the parent gas, through transfer reactions. Therefore $\mathrm{B}$ is reduced. The measurable consequence of this fact is that the gain increases with TMA concentration by factors up to $\times 16-50$ at $1-10$ bar, with the electric field remaining unchanged.

- when TMA further increases, B becomes larger, probably caused by the TMA molecule cooling down the electron swarm. Thus larger fields are required to achieve identical gas gains.

We conclude that, in Xe+TMA mixtures, a narrow operating range exists, where TMA is clearly advantageous over pure Xe for this kind of amplification structures. This occurs within a TMA concentration range of $\sim 1.4 \%-6.4 \%$ and $\sim 1.4 \%-2.5 \%$ at 1 and from 5 to 10 bar, respectively. It is known that quenched gases show a significantly reduced diffusion, improving the pattern recognition of pixelized readouts, as studied in [23].

\subsection{Varying the pressure}

In this section we present the results of gas gain and energy resolution obtained when the pressure was varied from 1 to 10 bar, using TMA concentrations within the range that was estimated as optimum in the previous section. Therefore, mixtures with TMA concentrations ranging from $1.5 \%$ to $2 \%$ were selected, and the values are specified in table 2 .

\begin{tabular}{c|c|c|c|c|c|c|c|c|c|c} 
Pressure & 1 & 2 & 3 & 4 & 5 & 6 & 7 & 8 & 9 & 10 \\
\hline \% TMA & 1.7 & 1.7 & 1.5 & 1.6 & 2.0 & 2.0 & 2.0 & 1.9 & 1.9 & 1.8
\end{tabular}

Table 2. TMA concentrations used for the systematic gas gain and energy resolution measurements when pressure was varied between 1 and 10 bar. Each TMA concentration is within the range that was considered as optimum for Penning transfer.

Gas gain curves are shown in figure 8 (left). It is noted that the amplification field necessary to reach any given gain increases with pressure, as already observed in pure Xe [13, [19]. The slope tends to decrease with pressure, thus at high pressures for a given change in the amplification field, the absolute change in gain is lower according to what has been observed in pure Xe and $\mathrm{Xe}+$ methane mixtures [19]. On the other hand, the maximum gain drops nearly exponentially with 
pressure for pressures above 2 bar, down to $\sim 400$ at 10 bar. However, the maximum gain at any pressure is still at least a factor 3 higher than for Micromegas operated in pure Xe [13]. This behaviour is probably caused by photons in the avalanche generated from molecular Xe excitations [19].
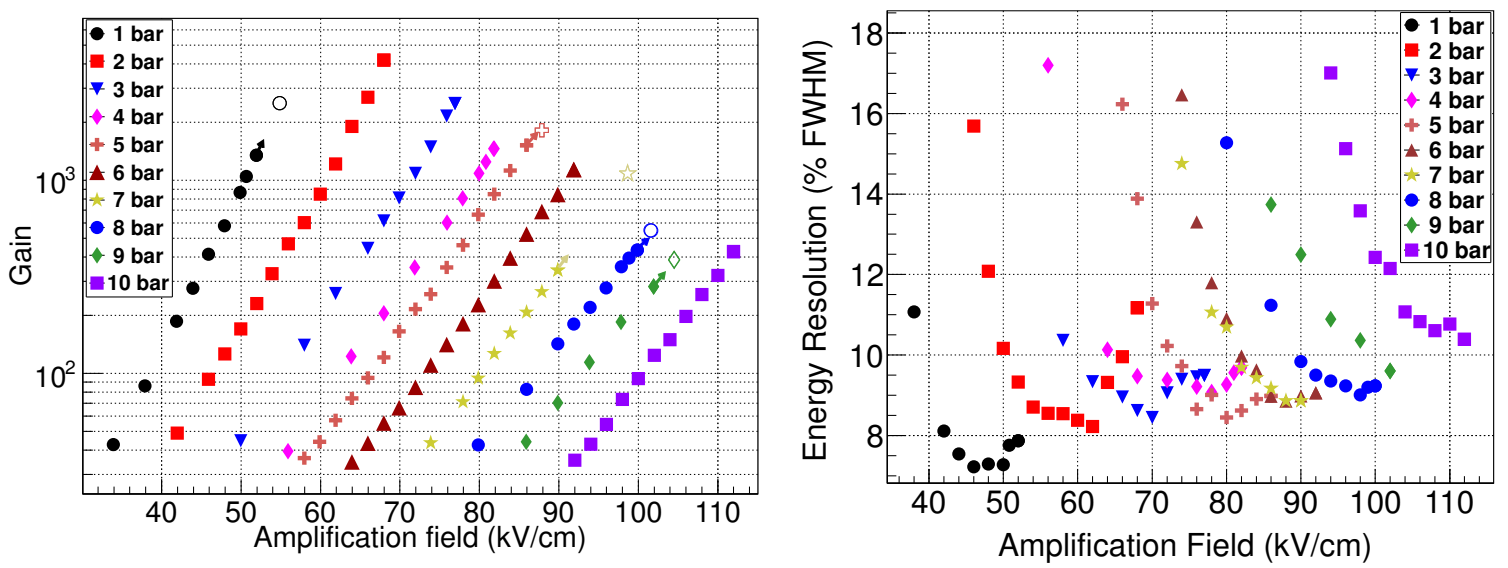

Figure 8. Dependence of the gain (left) and the energy resolution at $22.1 \mathrm{keV}$ (right) with the amplification field for a pressure ranging from 1 to $10 \mathrm{bar}$, at optimal TMA concentrations within the range $1.5 \%-2 \%$. An empty marker represents the maximum gain in case it was reached in a different mixture but with TMA concentration close to optimum values.

The energy resolution (Figure 8 , right) shows a rapid improvement with the amplification field, reaching a minimum value and then degrading at high fields. As mentioned before, the signal-tonoise ratio explains the high values at low fields, The best energy resolution measured at each pressure for gains within the range from 300 to 800 is shown in figure 9 (ם). A slight degradation with pressure is observed, which may be caused by electronegative impurities or inherent physical mechanisms [19]. Moreover, our deterioration in energy resolution with pressure has a tendency compatible with measurements performed by H. Sakurai et al [19], were the estimated level of impurities was below $3 \mathrm{ppm}$. Thus we may conclude that a mechanism inherent to the avalanche process in HP Xe+TMA mixtures is responsible for the observed degradation. As it has been noted, at higher pressure the reduced amplification field is weaker, a fact resulting in the increase of the number of excitations as compared to ionizations, hence more avalanche fluctuations and the subsequent degradation in the energy resolution [19, 22]. We plan to address this statement more quantitatively in future works.

The results of this work are compared in figure 9 with previous measurements of Micromegas detectors in pure Xe: with the same setup used in this study $(\boldsymbol{\Delta})$ [24] and with a different one ( $\mathbf{\nabla})$ [13]. The energy resolution achieved at $22.1 \mathrm{keV}$ is substantially better in this work, going down to $7.3 \%(9.6 \%)$ FWHM at 1 (10) bar. This fact translates into an improvement of a factor 2 (3) at 1 (10) bar as compared to previous measurements in pure Xe. Therefore, we can infer that the addition of TMA to Xe reduces the avalanche fluctuations; likely due to transfer reactions from the Xe excited states to TMA molecules (Penning effect).

Figure 10 shows the value of $(\ln (\ln (G) / P))$ as a function of $\left(S^{-1}\right)$, including data from all 


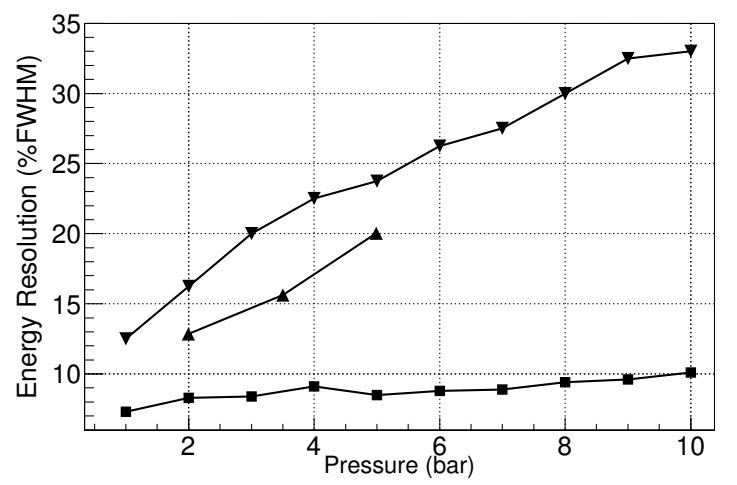

Figure 9. The dependence of energy resolution with pressure at $22.1 \mathrm{keV}$, for data from [13] $(\mathbf{\nabla})$, data [24] $(\boldsymbol{\Lambda})$ and in this work (ם).

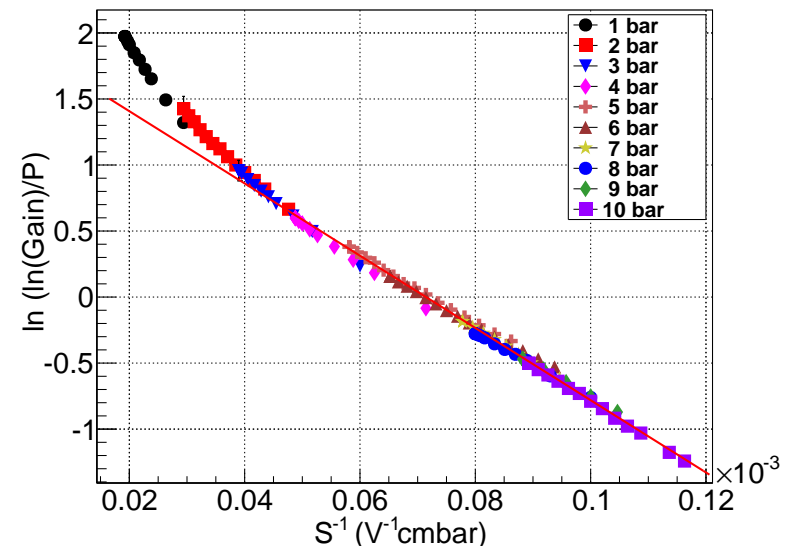

Figure 10. The natural logarithm of the reduced gas gain as a function of the inverse of the reduced amplification field. The red line represents a linear fit made for all data above 5 bar, which emphasizes the deviations from the expected behaviour for data below this pressure.

pressures measured. Under the assumptions implicit in the model of equations 4.1 and 4.2 we would expect all data points falling on the same straight line. However, deviations from the model expectation are observed for pressures below 5 bar, something that is more clearly observed in figure 11, where the parameters $A$ (left) and the ratio $B / A$ (right) are plotted as a function of the pressure. Both $A$ and $B$ were determined from linear fits of the curves showed in figure 10. As seen, $A$ drops with increasing pressure up to 5 bar, remaining at a relatively constant value for higher pressures, while $B / A$ increases with pressure, reaching a stable value from 5 bar on. In contrast, measurements performed in cylindrical proportional detectors in the same pressure range, both in pure $\mathrm{Xe}$ and in Xe-methane mixtures [19], as well as measurements performed with Micromegas up to 2.5 bar in Xe-methane [21] have shown that the parameters $A$ and $B$ are independent of the pressure, and thus the model of equations 4.1 and 4.2 represents a good description of those mixtures. We tentatively attribute the departure of our data from the expected model trends to the presence of Penning effect. However this fact needs further study, in particular using detailed Monte Carlo simulation of the avalanche microphysics, study that we expect to carry out in the 
near future.
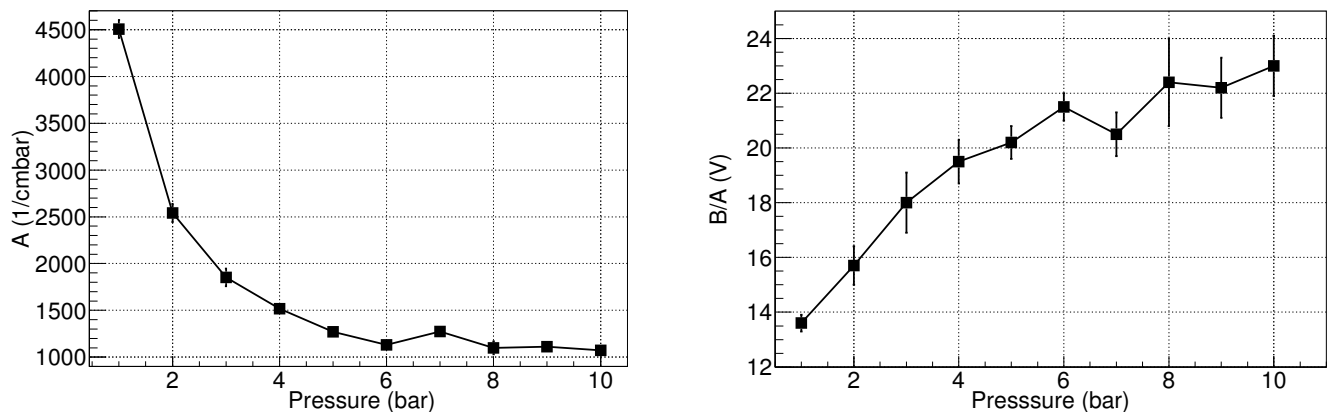

Figure 11. Parameter A (left) and the ratio of $B$ to $A$ (right) as a function of pressure. Parameters have been obtained from gas gain curves at each pressure, considering a semi-empirical formula of the first Townsend coefficient.

\section{Conclusions}

We have operated microbulk Micromegas detectors in high pressure Xe+TMA Penning gas mixtures, obtaining very good performance in terms of stability, gain and energy resolution. Systematic data on gain and energy resolution have been presented for a variety of TMA concentrations ranging from $0.3 \%$ to $15 \%$ and for gas pressures from 1 to 10 bar. We found that for TMA concentrations in the 1.5\%-2.5\% range, the Penning effect is maximum, requiring operating amplification fields at least $40 \%$ lower than pure Xe for the same gain, or gains up to a factor $\sim 100$ higher for the same amplification field. Maximum workable gains are superior by a factor at least $\times 3$ with respect to pure Xe, obtaining in particular gains above 400 at 10 bar.

For appropriate TMA fractions, energy resolutions down to $7.3 \%$ (9.6\%) FWHM at 1 (10) bar for the $22.1 \mathrm{keV}{ }^{109} \mathrm{Cd}$ peak could be achieved, an improvement of about a factor 2 (3) with respect to values obtained by microbulks in pure Xe [13]. This extrapolates into an energy resolution of $0.7 \%$ (0.9\%) FWHM at the $Q_{\beta \beta}$ value of Xe for 1 (10) bar, and therefore opens very good prospects for double-beta decay experiments.

In general, the performance of the detector is comparable to optimum values typically obtained with benchmark Micromegas mixtures (e.g. optimized Ar-isobutane mixtures). This result proves that Xe+TMA may be a mixture of choice for Micromegas in applications envisaging the use of $\mathrm{Xe}$ as conversion gas, especially at high pressures.

\section{Acknowledgments}

We are grateful to our colleagues of the groups of the University of Zaragoza, CEA/Saclay and our colleagues from the NEXT and RD-51 collaborations for helpful discussions and encouragement. We thank R. de Oliveira and his team at CERN for the manufacturing of the microbulk readouts. We acknowledge support from the European Commission under the European Research Council T-REX Starting Grant ref. ERC-2009-StG-240054 of the IDEAS program of the 7th EU Framework Program. We also acknowledge support from the Spanish Ministry of Economics and 
Competitiveness (MINECO), under contracts ref. FPA2008-03456 and FPA2011-24058, as well as under the CUP project ref. CSD2008-00037 and the CPAN project ref. CSD2007- 00042 from the Consolider-Ingenio 2010 program of the MICINN. Part of these grants are funded by the European Regional Development Fund (ERDF/FEDER). F.I. acknowledges the support from the Eurotalents program and D.C.H. of the Univ. Zaragoza, under the program PIF-UZ-2009-CIE-03.

\section{References}

[1] R. Luescher et al, Search for $\beta \beta$ decay in ${ }^{136}$ Xe: new results from the Gotthard experiment, Phys. Letters B 434 (1998) 407.

[2] V. Alvarez et al., NEXT-100 Technical Design Report (TDR). Executive summary, 2012 JINST 7 T06001.

[3] Auger et al, Search for Neutrinoless Double-Beta Decay in ${ }^{136}$ Xe with EXO-200, Phys. Rev. Lett. 109 (2012) 032505.

[4] Y. Giomataris, P. Rebourgeard, J. Robert and G. Charpak, MicrOMEGAS: a high-granularity position-sensitive gaseous detector for high particle flux enviroments, Nucl. Instrum. Meth A 376 (1996) 29.

[5] I.G. Irastorza et al., Status of $R \& D$ on Micromegas for Rare Event Searches: The T-REX project, EAS Publications Series 53(2012) 147.

[6] S. Andriamonje et al., Development and performance of Microbulk Micromegas detectors, 2010 JINST $5 \mathrm{P} 02001$.

[7] S. Cebrian et al., Radiopurity of micromegas readout planes, Astropart. Phys 34 (2011) 354 .

[8] T. Dafni et al., Energy resolution of alpha particles in amicrobulk Micromegas detector at high pressure argon and xenon mixtures, Nucl. Instrum. Meth.A 608 (2009) 259.

[9] Y. Giomataris, Development and prospects of the new gaseous detector "Micromegas", Nucl. Instrum. Meth. A 419(1998) 239.

[10] G.D. Alkhazov, Statistics of electron avalanches and ultimate resolution of proportional counters, Nucl. Instrum. Meth. A 89 (1970) 155.

[11] S. Andriamonje et al., Development and performance of microbulk micromegas detectors, 2010 IINST 5 P02001.

[12] S. Cebrián et al., Micromegas readouts for double beta decay searches, JCAP 10 (2010) 010.

[13] C. Balan. et al., Micromegas operation in high pressure xenon: charge and scintillation readout, 2011 IINST 6 P02006.

[14] A. Calcaterra et al., A new concept for streamer quenching in resistive plate chambers, Nucl. Instrum. Meth. A 565(2006) 444.

[15] B. Ramsey and P. Agrawal, Xenon- based Penning mixtures for proportional counters, Nucl. Instrum. Meth. A 278 (1989) 576 .

[16] D. Nygren., Can the intrinsic energy resolution in xenon be surpassed, J. Phys.: Conf. Ser. 309 (2011) 012006 .

[17] F.J. Iguaz et al, Characterization of microbulk detectors in argon-and neon-based mixtures, 2012 IINST 7 P04007 
[18] K. White et al., Gas gain in xenon +2,3 dimethyl-2-butene filled proportional counters, Nucl. Instrum. Meth. A 260(1987) 443 .

[19] H. Sakurai et al., High pressure xenon proportional counter up to 10 bar, Nucl. Instrum. Meth. A 307 1991504

[20] F. Sauli, Principles of operation of multiwire proportional and drift chambers, CERN-77-09 (1975).

[21] P.K. Lightfoot et al., Development of a double-phase Xenon cell using micromegas charge readout for applications in dark matter physics, Nucl. Instrum. Meth. A 554 (2005) 266.

[22] H. Sakurai and B.D. Ramsey, Dependence of energy resolution on anode diameter in xenon proportional counters, Nucl. Instrum. Meth. A 313 (1992) 155.

[23] S. Cebrian et al., Background discrimination in a high pressure Xenon Micromegas TPC for a double beta decay experiment on ${ }^{136} \mathrm{Xe}$, article in preparation.

[24] T. Dafni on behalf of the NEXT Collaboration, Micromegas planes for the neutrinoless double beta decay search with NEXT, J.Phys.: Conf. Ser 309 (2011) 012009 